\title{
Desarrollo del pensamiento estadístico en estudiantes de nivel superior a través de una Experiencia Educativa
}

\section{Statistical thinking development in superior level students through one educative experience}

\author{
Diana Del-Callejo-Canal ${ }^{1}$ \\ Margarita Canal-Martínez ${ }^{2}$ \\ Mónica Rubiette Hákim-Krayem ${ }^{3}$
}

\begin{abstract}
Resumen: La finalidad de este artículo es reflexionar sobre los resultados que se observan como consecuencia de la implementación de las estrategias para desarrollar un pensamiento estadístico en estudiantes universitarios, a través de la Experiencia Educativa titulada "¿Cómo aplicar la estadística en proyectos de investigación?" La perspectiva de los dominios o competencias adaptadas de Garfield, DelMas y Chance (2003) integran el marco teórico. Se utilizaron las evidencias de aprendizaje de 40 estudiantes en tres periodos escolares; la recolección de datos se realizó mediante las producciones escritas por ellos, en dos momentos: al inicio y al finalizar el curso. Los resultados muestran que $40 \%$ de los estudiantes logró aplicar la técnica correcta, sin dificultades para argumentar por escrito y oralmente sus hallazgos. Los resultados de esta experiencia pueden permitir a profesores de estadística tomar decisiones sobre su planeación didáctica.
\end{abstract}

Fecha de recepción: 7 de septiembre de 2018. Fecha de aceptación: 6 de marzo de 2020.

1 Instituto de Investigaciones y Estudios Superiores Económicos y Sociales. Universidad Veracruzana, ddelcallejo@uv.mx, orcid.org/0000-0003-4753-6577.

2 Instituto de Investigaciones y Estudios Superiores Económicos y Sociales. Universidad Veracruzana, mcanal@uv.mx, orcid.org/0000-0002-1258-5902.

${ }^{3}$ Instituto de Investigaciones y Estudios Superiores Económicos y Sociales. Universidad Veracruzana, rhakim@uv.mx, orcid.org/0000-0003-0841-397X. 
Palabras clave: Pensamiento estadístico, conceptualización estadística, competencias estadísticas, educación estadística, reporte estadístico.

\begin{abstract}
The purpose of this article is to analyze if the results of the implementation of strategies to development a statistical thinking skill in the educative experience for bachelor students entitle How applied the statistics on a research project? The concept and perspective adapted of Garfield, DelMas and Chance (2003) integrated the theorical framework. We use the learning evidences of 40 students during three school periods, through the data recollection of the writing production in two moments: before the course and after them. We found that $40 \%$ of the students applied the right statistical technique and write and expose theirs results. The founds of this experience can allow the professors make decisions for the didactic planning.
\end{abstract}

Keywords: Statistical literacy, Statistical conceptualization, Statistical skills, Statistical education, statistical report.

\title{
1. INTRODUCCIÓN
}

Uno de los retos actuales en el campo de la Educación, es sin duda la responsabilidad social que los facilitadores tenemos ante los estudiantes, manifiesto que se declara en el Modelo Educativo Integral y Flexible (MEIF) de la Universidad Veracruzana (UV), que además concibe la necesidad de realizar innovaciones planeadas, organizadas y concretas para que a través de la práctica docente y en su aplicación con los estudiantes, se logre el desarrollo de las competencias -habilidades cognitivas en estudiantes universitarios para resolver problemas de su contexto real- que generen nuevas formas de ser-hacer y convivir en sociedad (Universidad Veracruzana, 1999).

Resulta pertinente aclarar que, todos los programas educativos de nivel licenciatura de la UV se estructuran por áreas de formación: Básica general, Iniciación a la disciplina, Disciplinar, Terminal y Elección libre (AFEL); nuestra propuesta se inscribe en esta última. El AFEL, está dirigida a una formación complementaria del desarrollo integral de los estudiantes (Universidad Veracruzana, 1999), y por lo tanto permite a los estudiantes de cualquier programa educativo elegir dentro de un catálogo de Experiencias Educativas (EE), algunas 
de ellas. En dicha área, encontramos una brecha de oportunidad para innovar y fomentar ambientes de aprendizaje inter y multidisciplinarios para aplicar la estadística en la resolución de problemas de la vida real, desde diferentes áreas del conocimiento y disciplinas, enriqueciendo así la formación integral de los futuros profesionistas y coadyuvar en el desarrollo de las competencias de los estudiantes por medio de intervenciones pedagógicas como lo es la innovación, implementación de estrategias didácticas, de recursos y métodos de enseñanza, elaboración de materiales, etcétera.

Desde este hilo conductor decidimos diseñar e implementar la EE denominada ¿Cómo aplicar la estadística en proyectos de investigación?, con la finalidad de proporcionar una EE inter y multidisciplinaria que ligara a la estadística con la investigación. Además, dentro de las competencias de las matemáticas (asignatura presente en los programas educativos), la estadística ocupa un lugar esencial, pues es la disciplina, que permite recolectar, clasificar, analizar e interpretar información cuantitativa para atender problemáticas en diferentes contextos y situaciones y de forma crítica y autónoma (Rouquete, Suárez y Ariza, 2014).

Sin embargo, en nuestra experiencia como docentes, observamos que los estudiantes de licenciatura, a pesar de haber cursado por lo menos trece años de educación de matemáticas (SEP, 2011, 2016, 2017), no han logrado desarrollar la competencia de análisis de situación, argumentación y razonamiento matemático y mucho menos el desarrollo del pensamiento estadístico, procesos cognitivos que se requieren para la comprensión conceptual de la estadística.

Aunado a lo anterior, consideramos que actualmente la cantidad de datos es abrumadora, aprender a tomar decisiones informadas basadas en los datos disponibles y confiables, con al menos las bases estadísticas pertinentes, es vital no solo para investigadores o profesionales de la estadística, sino para los ciudadanos en general (Franklin, Bargagliotti, Case, Kader, Scheaffer y Spangler, 2015 y Weiland, 2017).

En la década de 1980, varios grupos reconocidos de educación en las matemáticas como, por ejemplo: National Council of Teahers of Mathematics (NCTM) o National Assesment of Educational Progress (NAEP), propusieron a la educación estadística como una competencia necesaria en los graduados de educación media para prosperar en el mundo moderno (Franklin, Kader, Peck, Perry y Scheaffer 2005). A pesar de la necesidad detectada de incluir a la estadística como una asignatura ineludible en los niveles de educación superior, no se ha logrado desarrollar una pertinente comprensión conceptual estadística. Tu y 
Snyder (2017), comentan que los estudiantes en los cursos de estadística regularmente carecen de habilidad para generar razonamiento y pensamiento estadístico.

La educación estadística no ha sido lo suficientemente accesible, ya sea como menciona Brown y Kass (2009), porque el currículo de las materias o cursos en estadística se basa en nociones no actualizadas o bien porque la preocupación en la enseñanza de la estadística se enfoca en el uso de las tecnologías y paqueterías estadísticas, que si bien es una herramienta útil, porque agiliza los procesos operativos, no refuerza en la profundización de los conceptos teóricos incluyendo en ellos el desarrollo del pensamiento estadístico (Gould, 2010).

De allí que la EE implementada -por tres periodos escolares consecutivos con estudiantes de diversos programas educativos (PE)- aprovecha la relación natural existente entre la estadística y la investigación, cuya finalidad es promover en los estudiantes un pensamiento estadístico útil para conjeturar e inferir, a través de las estrategias del método científico, la habilidad de explicar un fenómeno real con herramientas estadísticas adecuadas y sean capaces de incorporarse al mercado laboral para ser productivos y adaptarse a los cambios vertiginosos actuales con capacidad crítica y creadora (Rouquette, Suárez y Ariza, 2014). En el diseño curricular de esta EE, planteamos como unidad de competencia que el estudiante conozca, analice y aplique las herramientas estadísticas en proyectos de investigación, mediante estrategias metodológicas y teóricas pertinentes.

Al plantear y reflexionar sobre la pregunta de investigación ¿Qué resultados se observan como consecuencia de la implementación de las estrategias diseñadas para desarrollar el pensamiento estadístico? nuestra finalidad-aparte del logro del objetivo planteado en la EE- es conocer la capacidad para explicar, interpretar y emitir juicios a fenómenos de la realidad y para la toma de decisiones adecuadas con responsabilidad y una actitud crítica y obtener con ello, la conceptualización estadística, desde el logro del pensamiento estadístico.

Escribir este artículo compartiendo nuestros hallazgos, tiene dos intenciones: 1) hacer un ejercicio de autoevaluación que pueda servir a otros docentes para enriquecer su práctica docente, y 2) compartir los resultados de una comprensión integradora de la estadística, más allá de plantear ejercicios diseñados específicamente para probar su eficacia a partir de número de aciertos o fallos de un grupo de estudiantes. Esta comprensión integradora requirió identificar claramente cuáles eran las técnicas y herramientas estadísticas necesarias para 
analizar cualquier problema de investigación que el estudiante pudiera plantear y con ello, propiciar un pensamiento estadístico lo que sería un aporte innovador a la educación estadística.

\section{MARCO CONCEPTUAL: PENSAMIENTO ESTADÍSTICO Y COMO PROVOCARLO}

La sociedad demanda profesionistas con competencias, habilidades y actitudes de investigación estadística que puedan apoyar con propuestas pertinentes a la solución de problemáticas de diversa índole, pero también y como ya se mencionó, se requiere que desarrollen las competencias para manejar el bagaje de datos que en la actualidad existen, lo que contrapone a la forma tradicional de visualizar a la estadística únicamente como el manejo de meros cálculos numéricos.

Es importante desarrollar el pensamiento estadístico de los estudiantes, ya que hoy en día estamos expuestos a información estadística todo el tiempo, en Medicina, en Publicidad, en Ciencias Sociales, Política, Economía, Ecología, incluso en la información que recibimos a través de las aplicaciones sociales como facebook, ¿Quién de nosotros no ha leído titulares como: una copa de vino al día puede disminuir la probabilidad de un infarto? Compartimos la información, sin cuestionar la veracidad de los estudios y la asumimos como cierta, porque no nos sentimos capacitados para discutirla. Pues bien, el desarrollo del pensamiento estadístico nos aleja de esas explicaciones mágicas, y nos induce a evaluar los argumentos estadísticos de los demás y sustentar los propios (Tunstall, 2018).

A pesar de su importancia para la formación de ciudadanos responsables, el pensamiento estadístico no es una habilidad que se explicite como competencia transversal en los cursos en matemáticas o estadística (Tunstall, 2018). Además, de que dicha habilidad no se puede desarrollar únicamente a partir de ejercicios estandarizados.

Ante lo anterior, en nuestra búsqueda de cómo lograr ese pensamiento estadístico, y debido a que es una actividad intelectual de múltiples aspectos y que se desarrolla gradualmente, encontramos que el desarrollo de una comprensión conceptual estadística requiere de competencias y habilidades. Garfield, et al., 2003, conceptualizan dominios o competencias adaptadas como son: alfabetización estadística que refiere a las habilidades básicas sobre conceptos, 
vocabularios y simbología necesarios para discernir sobre la información o resultados de investigación como, por ejemplo: organizar, presentar y construir tablas de datos para diferentes representaciones de los mismos; el razonamiento estadístico que consiste en que los estudiantes sean capaces de deducir el porqué de la aplicación de la estadística en el uso y proceso de la información, esto incluye hacer interpretaciones de los datos, resumir información, conectar conceptos e incluso combinar ideas para obtener resultados más adecuados; y el pensamiento estadístico que involucra el entendimiento de por qué y cómo la estadística puede ser aplicada en casos reales, de generar preguntas, colectar datos y seleccionar el análisis adecuado para responderla, ejecutar, hacer juicios de valor, de criticar y evaluar los resultados de un problema resuelto con las técnicas y herramientas estadísticas, lo que significa que hay una reflexión sobre el uso apropiado de la estadística para hacer inferencias confiables y pertinentes y que éstas sean evidenciadas a través de la comprensión del proceso integral de una investigación.

Y además, que tengan la oportunidad de dar sentido a sus conjeturas relacionadas con su disciplina con objetividad; explorar sus propias ideas; plantear hipótesis a partir de datos que sirven de base para una investigación y adaptar estrategias para argumentar sus conclusiones a través del uso adecuado de la estadística.

En conclusión, la finalidad de esta intervención pedagógica es provocar que los estudiantes desarrollen el pensamiento estadístico, a través de un aprendizaje gradual, partir de los conceptos y simbología básicos de la estadística (alfabetización estadística), deducir el porqué de la aplicación de la estadística en el uso y proceso de la información (razonamiento estadístico) y lograr que sean capaces de evidenciar su desempeño a través de la demostración y aplicación de los conocimientos y habilidades necesarias para analizar e interpretar y proponer alternativas de solución a problemáticas de su entorno, generándose aún más preguntas a futuro (pensamiento estadístico).

Para responder a nuestra pregunta de investigación y suscitar el logro del pensamiento estadístico, consideramos que el contexto juega un rol trascendental: Ios datos estadísticos provienen de un contexto y el modelo y su interpretación tiene que estar inmerso en ese contexto (Kuntze, Aizikovitsh-Udi y Clarke, 2017). Aspecto que va con lo establecido en el proyecto GAISE (Guidelines for Assessment and Instruction in Statistics Education), propuesto por American Statistical Association (Franklin et al., 2005) y otras acotaciones recientes de GAISE College Report ASA Revision Committee, 2016, como son: 
1. Fomentar el aprendizaje del pensamiento estadístico en el estudiante, como un proceso de investigación que busque resolver un problema de la disciplina que estudia y tome decisiones.

2. Focalizar el alcance conceptual (más allá del mero conocimiento procedimental).

3. Integrar datos reales en un contexto y con un propósito (aprendizaje activo).

4. Usar la tecnología como apoyo para desarrollar conceptos y analizar los datos.

5. Usar la evaluación con el fin de mejorar el aprendizaje de los estudiantes, identificando errores y aciertos y, por ende, retroalimentar y mejorar la función docente.

\section{METODOLOGÍA}

Con el objetivo de conocer los resultados de la implementación de las estrategias planteadas durante el periodo escolar de aplicación para desarrollar el pensamiento estadístico, se consideraron dos instrumentos de medición: a) Se aplicó un cuestionario (antes y después del curso) en el que se le solicitó al estudiante que describiera con sus palabras el uso de la estadística en proyectos de investigación; se recopiló la información en una tabla de datos para comparar posteriormente. Se valoró el número de palabras que ocupó el estudiante para describir lo que se le pidió y se realizó un análisis de texto en la paquetería $R$-project, versión 3.5.0; b) Un reporte estadístico en formato de artículo científico, para evaluar lo aprehendido sobre el uso adecuado de las técnicas y herramientas estadísticas, así como la comprensión de la presentación de los resultados de una manera académicamente válida (competencias adquiridas y redacción correcta, orden en las ideas escritas, formas de citado correctas, etc.), además de la presentación oral en clase sobre el reporte estadístico generado por el estudiante destacando el uso de la metodología estadística en términos de un pensamiento estadístico.

\subsection{Programa de LA EE}

La EE ¿Cómo aplicar la estadística en proyectos de investigación? Como propuesta educativa para la enseñanza-aprendizaje de la estadística, resalta la importancia de visualizar a la metodología estadística como un eje pertinente aplicable a cualquier disciplina. 
El programa de la EE que se oferta a través del AFEL, considera un máximo de 20 estudiantes - de cualquier disciplina-, para asegurar que el estudiante tenga una atención personalizada en su formación. Está estructurado en un total de 60 horas al semestre, con un valor de 6 créditos y es de carácter intersemestral (verano e invierno). Contiene tres temas centrales: 1) metodología estadística para proyectos de investigación -12 horas-; 2) técnicas y herramientas estadísticas para proyectos de investigación -32 horas-; y 3) presentación de reportes de investigación -16 horas-, temas que se van adecuando según lo observado en los cursos ya impartidos anteriormente.

Así mismo, dado que se permite la inscripción de estudiantes de cualquier programa educativo y disciplina, y para cumplir con el objetivo declarado en el programa de la $\mathrm{EE}^{4}{ }^{4}$ se diseñaron las siguientes estrategias: 1) una exploración de saberes previos de los estudiantes sobre estadística; 2) reajuste y elección de contenidos en los temas centrales, incluimos técnicas y herramientas esenciales de estadística exploratoria, por ejemplo, las medidas de tendencia central y de dispersión, para posteriormente introducir a los estudiantes a comparaciones y relaciones estadísticas, según el tipo de variables cualitativas o cuantitativas que se usen, entre otras; y 3) se elige como trabajo académico la elaboración de un reporte estadístico -evidencia del desempeño del estudiante al final del curso-, estructurado con la aplicación de lo aprendido en clase a un objeto de estudio (problema real) y definida la pregunta de investigación.

\subsection{DINÁMICA EN CLASE}

Una vez que se define el trabajo de investigación que evidencia los dominios de los estudiantes, las dinámicas y estrategias de enseñanza-aprendizaje que se utilizaron al interior del aula fueron pensadas y planeadas alrededor del mismo. En los primeros días, de manera intuitiva, el estudiante define su tema y la pregunta de investigación. Se les proporciona el acceso a repositorios de datos como Web of science, donde inicia la primera búsqueda académica para seleccionar un artículo que sea algo similar a lo que pensó o le interesó. Posteriormente y conforme avanza el curso, el estudiante es capaz de construir una pregunta de investigación lo suficientemente clara como para identificar y definir las variables

\footnotetext{
4 Que el estudiante aplique la estadística en proyectos de investigación mediante el uso teórico-metodológico de la misma, para explicar e interpretar un fenómeno de la realidad.
} 
que usará, y si lo que le interesa es comparar o relacionar objetos de estudio, la pregunta le servirá para plantear una hipótesis estadística.

Dependiendo del trabajo de investigación elegido, los estudiantes diseñan instrumentos y levantan encuestas piloto (fuentes primarias) o bien, obtienen y hacen uso de los datos de alguna fuente oficial (fuentes secundarias). A través del diálogo grupal y ejemplos prácticos del uso de la estadística para identificar diferencias entre variables cualitativas y cuantitativas, el estudiante selecciona el tipo de variables para su proyecto.

Las técnicas estadísticas analizadas en clase se explican bajo tres enfoques: ¿Qué es la técnica?, ¿Cómo opera (procedimiento) la técnica? y ¿Para qué sirve la técnica? Los ejercicios se realizan en clase utilizando el software R-project. Al término de esta fase, la siguiente actividad del estudiante es reflexionar y cuestionarse: ¿Qué de lo visto en clase me sirve y puedo aplicarlo a mi trabajo de investigación?

\subsection{PARTICIPANTES Y TRATAMIENTO}

A la fecha, la EE se ha ofertado y aplicado durante tres periodos intersemestrales: diciembre 2017-enero 2018 (invierno), junio-julio de 2018 (verano) y enero 2019 (invierno). Formalmente han cursado un total de 40 estudiantes.

A los estudiantes se les impartió un curso de 60 horas -por cada periodo-, cuya meta final es que, con lo aprendido, sean capaces de: 1) aplicar la técnica estadística correcta para resolver su pregunta de investigación, 2) escribir con claridad el por qué seleccionaron esa metodología, los resultados a los que llegaron y las conclusiones y, 3) explicar de manera oral sus hallazgos. Con la evidencia de dichos puntos, se está cumpliendo con el objetivo del programa de la EE, así mismo, se evidencia el desarrollo del pensamiento estadístico, planteado como finalidad de este artículo.

\subsection{EVALUACIÓN DEL DESEMPEÑO}

La acreditación de esta EE del AFEL, requiere cuando menos $60 \%$ de evidencias entregadas en tiempo y forma y $80 \%$ mínimo de asistencias. Para asignar un puntaje a las evidencias de desempeño de los estudiantes, se determinaron 
criterios en una escala del 1 al 10 de acuerdo a las normas de acreditación de control escolar de la UV, establecido al inicio del curso:

- Calificación de 5 (no acreditado): Si el estudiante no logra la habilidad de relacionar su tema y pregunta de investigación con la metodología estadística vista en clase y, tampoco explica de manera escrita y oral sus finalidades y resultados, se asume que no ha logrado desarrollar los conocimientos mínimos necesarios para declarar un incipiente aprendizaje estadístico. Además de ello, se suma el incumplimiento en la entrega de evidencias durante el curso e inasistencias.

- Calificación 6 a 7. Alfabetización estadística. Si el estudiante logra el dominio básico de relacionar su tema y pregunta de investigación con los conceptos, vocabulario y simbología estadística; es capaz de presentar, organizar y construir tablas de datos y/o representaciones gráficas y determinar de manera incipiente qué técnica estadística podría aplicar, pero tiene dificultades para argumentar el procedimiento estadístico, así como, escribir y explicar oralmente sus hallazgos.

- Calificación 8. Razonamiento estadístico. Si el estudiante logra el dominio de relacionar su tema y pregunta de investigación con la selección e interpretación de datos, ordenar y resumir información; combina ideas para obtener resultados más adecuados; conecta conceptos e incluso combina ideas para obtener resultados idóneos; selecciona y aplica la técnica estadística correcta. Pero, presenta dificultad en deducir el porqué del uso de ésta en la interpretación del tema y resolución de su pregunta, y no logra hacer juicios de valor basados en criterios específicos, con organización, con lenguaje adecuado.

- Calificación 9 a 10. Pensamiento estadístico. Si el estudiante logra el dominio de relacionar su tema y pregunta de investigación con: disertar por qué y cómo aplicar la técnica estadística correcta; definir, colectar, organizar y valorar qué información es pertinente; generar preguntas avanzadas que orienten su proceso estadístico; sin dificultades para escribir y explicar oralmente sus hallazgos y resultados, interpretando con argumentos, coherencia y claridad; hacer inferencias confiables y pertinentes; y que exista evidencia de la relación en cuanto al proceso integral del uso de la estadística y la investigación. 


\section{RESULTADOS}

Hemos registrado 16 programas educativos de procedencia de los 40 estudiantes que a la fecha han cursado la EE, principalmente de Economía, Ingeniería electrónica, Estadística, Biología y algunos del área de Ciencias de la Salud (tabla 1).

Tabla 1. Frecuencias de alumnos inscritos a la EE, por carrera de procedencia.

\begin{tabular}{lr}
\hline Carrera & Frec. Rel. \\
Nutrición & $3 \%$ \\
Contaduría & $3 \%$ \\
Publicidad y relaciones p. & $3 \%$ \\
Ingeniería ambiental & $3 \%$ \\
Ingeniería en alimentos & $3 \%$ \\
Ingeniería química & $3 \%$ \\
Informática & $3 \%$ \\
Ingeniería Mecánica & $3 \%$ \\
Química clínica & $3 \%$ \\
Psicología & $5 \%$ \\
Administración & $5 \%$ \\
Químico Farmacéutico Biólogo & $8 \%$ \\
Biología & $10 \%$ \\
Estadística & $15 \%$ \\
Ingeniería electrónica & $18 \%$ \\
Economía & $18 \%$ \\
\hline Total & $100 \%$ \\
\hline
\end{tabular}

Fuente: Elaboración propia.

De acuerdo con los resultados valoramos que la EE a través de la metodología utilizada, reporta:

- $7.5 \%$ de índice de reprobación. Estudiantes que no lograron relacionar y aplicar la técnica estadística correcta con la pregunta de investigación y por tanto tampoco lograron escribir, ni explicar oralmente sus hallazgos con claridad, aparte de que no entregaron los avances requeridos. 
- $20 \%$ de los estudiantes lograron aplicar la técnica correcta, pero tienen dificultades importantes para escribir y explicar oralmente sus hallazgos - alfabetización estadística-.

- $32.5 \%$ de los estudiantes logró aplicar la técnica correcta, con dificultades medias para escribir y explicar oralmente sus hallazgos -razonamiento estadístico-.

- 40\% de los estudiantes logró aplicar la técnica correcta, sin dificultades importantes para argumentar oralmente sus hallazgos - pensamiento estadístico- (figura 1).

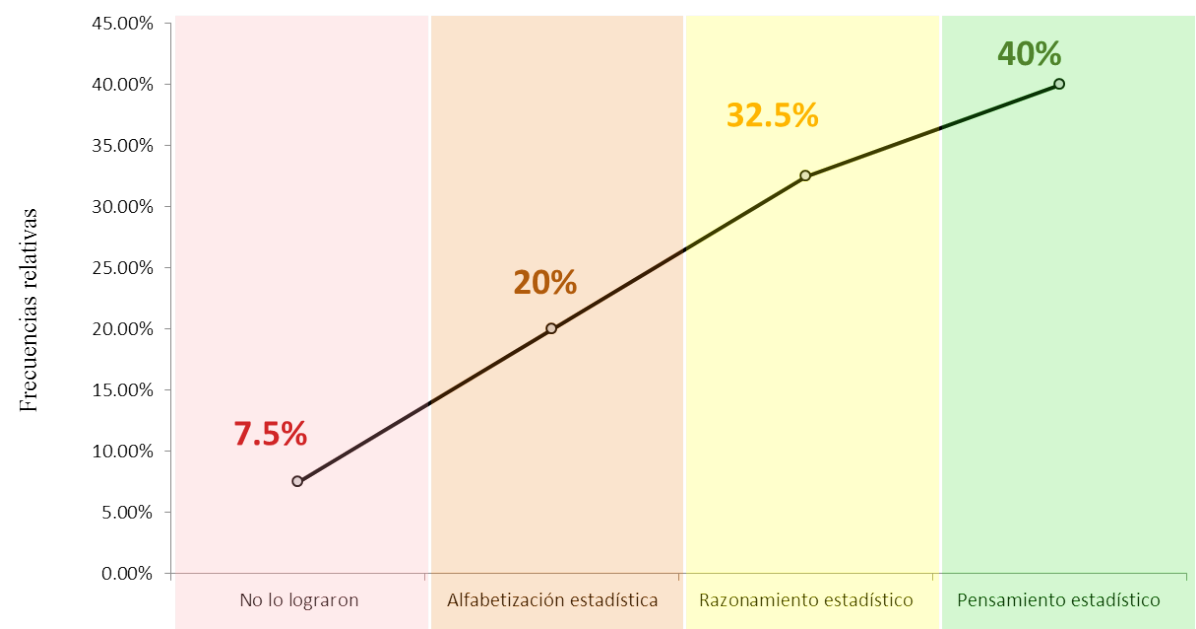

Figura 1: Frecuencias de calificaciones finales en los tres periodos ofertados Fuente: Elaboración propia.

Durante el desarrollo de la EE se fomentó un ambiente de respeto, diálogo, autocrítica y solidaridad con la finalidad de aprender de sí mismo y de los demás. Reconocemos que el error es una fuente de aprendizaje, por eso la retroalimentación de las actividades en clase y fuera de ella son fundamentales, ya que permite al estudiante percatarse de sus errores y de cómo corregirlos.

Durante las tres aplicaciones se ha observado que, a partir de la primera retroalimentación, los estudiantes ganan confianza y se involucran entre ellos, dando opiniones y consejos para mejorar sus proyectos; también han servido para darnos cuenta que, la principal dificultad de los estudiantes está en la elaboración de su pregunta de investigación y en la identificación y 
operacionalización de las variables. Esto lo hemos subsanado a través de dialogar y apoyarlos en la construcción de las preguntas e identificación de las variables necesarias de acuerdo con el tema elegido.

Consideramos que la elaboración del reporte estadístico a manera de un artículo científico es un acierto y es una de las evidencias más claras de la asimilación de los temas incluidos en el curso. Al inicio, ninguno de los estudiantes inscritos se consideraba a sí mismo en condiciones de construir un artículo científico y al finalizar $92.5 \%$ de ellos ha sido capaz de entregar uno.

Los resultados nos han permitido reconocer los aciertos y dificultades del curso, por ejemplo, debimos incluir el tema de: construcción de una tabla de datos; en la planeación original asumimos que la construcción de una tabla de datos no era necesaria, pero alrededor de 30\% de los estudiantes no han tenido experiencia con el traslado de la recopilación de datos a una tabla de datos estadísticamente funcional, así que reestructuramos para hacer un espacio para este tema. También ha sido necesario repasar sobre el método científico. Observamos que, a pesar de llevar cursos de metodología de investigación durante su formación disciplinaria, aproximadamente 80\% de los estudiantes no sabe cómo aplicarlo en la práctica.

\subsection{DOMINIOS O COMPETENCIAS}

\section{a) Conocimiento de la estadística declarado por los estudiantes antes y des- pués del curso de la $\mathrm{EE}$}

Los 40 estudiantes respondieron un instrumento antes y después del curso para valorar la asimilación del contenido de la EE. Entre otras cosas, describieron con palabras el uso de la estadística en proyectos de investigación. Como resultado del análisis de texto de esta instrucción, se observó que antes del curso el estudiante utilizó en promedio 8 palabras para describir el uso de la estadística en proyectos de investigación; mientras que, al finalizar el curso, fue capaz de expresarse en promedio con 31 palabras para responder la misma pregunta. Algunas de las respuestas más significativas, se sintetizan en la tabla 2. 
Tabla 2. Respuesta antes y después del curso a la instrucción "Describe con tus palabras el uso de la estadística en proyectos de investigación"

\begin{tabular}{cl}
\hline \multicolumn{2}{c}{ Describe con tus palabras el uso de la Estadística en proyectos de investigación } \\
\hline \multicolumn{1}{c}{ Antes de iniciar el curso } & \multicolumn{1}{c}{ Al finalizar el curso } \\
\hline Para saber la probabilidad de las cosas. & $\begin{array}{l}\text { La estadística se aplica para tener un mejor ma- } \\
\text { nejo de información en la investigación, ya que } \\
\text { esta estudia y presenta los datos de una manera } \\
\text { más accesible a entender. }\end{array}$ \\
\hline Para aceptar o rechazar una hipótesis de in- & $\begin{array}{l}\text { La estadística la podemos aplicar en distintas in- } \\
\text { vestigaciones en cualquier disciplina, las cuales } \\
\text { se basan en plantear hipótesis, seguir una meto- } \\
\text { dología, hasta llegar a los posibles resultados. }\end{array}$ \\
\hline Obtención de promedios, rangos y gráficos & $\begin{array}{l}\text { La estadística en un trabajo de investigación es } \\
\text { como la alimentación de un bebé, necesaria para } \\
\text { poder crecer y dependiendo de la edad (o dificul- } \\
\text { tad del trabajo) habrá un alimento adecuado } \\
\text { para él. }\end{array}$ \\
\hline Traducir datos de una forma cuantitativa. & $\begin{array}{l}\text { Creo que es una herramienta fundamental, la } \\
\text { cual si es usada de manera adecuada puede fa- } \\
\text { cilitar la manera de explicar los resultados. }\end{array}$ \\
\hline Determinar conjuntos. & $\begin{array}{l}\text { Al realizar investigación uno recopila o genera } \\
\text { datos. Las herramientas de la estadística nos per- } \\
\text { miten trabajar estos datos para poder sacar con- } \\
\text { clusiones de ellos. Es importante conocer la } \\
\text { naturaleza de lo que investigamos para poder } \\
\text { usar y escoger la mejor herramienta estadística. }\end{array}$ \\
\hline
\end{tabular}

Fuente: Elaboración propia.

En los gráficos de nube se observa un gran cambio. Mientras que antes del curso, el estudiante asoció el uso de la estadística en dos aspectos: datos e hipótesis. Al final del curso, el estudiante relacionó a la estadística con investigación, herramienta, resultados, adecuada, importante, mejor. Esto evidenció el avance significativo en la comprensión y sentido del uso de la estadística en un proyecto de investigación y por lo tanto en el desarrollo de su pensamiento estadístico (ver figura 2). 
Uso de palabras para describir el uso de la Estadística antes del curso

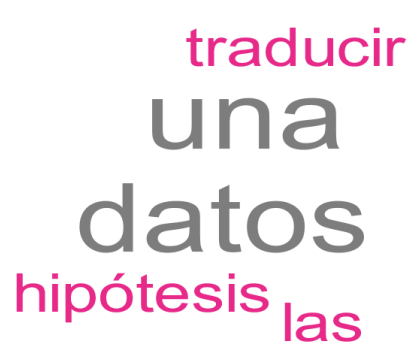

Uso de palabras para describir el uso de la Estadística después del curso.

Figura 2. Análisis de texto antes y después del curso.

Fuente: Elaboración propia con base al cuestionario antes/después. Paquete R-project versión 3.5.0.

\section{b) Reporte estadístico y el tipo de preguntas que genera}

Encontrar el ejercicio que une estas dos temáticas: metodología de investigación y estadística es el reto más grande dentro de esta EE. La alternativa que encontramos fue que, el estudiante decida un tema de investigación en total libertad y agrado. En consecuencia, los ejercicios diseñados de la clase no son estandarizados, ni tampoco tomados de un libro, esto creemos es lo que hace distinta esta experiencia.

Las temáticas que se han abordado en el curso son variadas, por mencionar algunas: "Path Planning, para robots con redes resistivas, ocupando como método de búsqueda el algoritmo LCC"; "Diseño y construcción de un prototipo para el tratamiento de residuos orgánicos de piña, papaya y plátano en 21 días"; "El efecto de la crisis del euro en el consumo de productos provenientes de América Latina"; "Diversidad de cnidarios en el arrecife rocoso de la Mancha, Veracruz". La diferencia de temas en los que se puede aplicar una misma técnica estadística otorga un valor agregado a esta innovación educativa y provoca en los estudiantes la curiosidad e interés investigativo.

El trabajar tablas provenientes de datos reales, provoca que en muchas ocasiones se planteen dudas que no surgen con los ejercicios tradicionales, ni se encuentran las respuestas en ningún libro, a estas las hemos Ilamado preguntas avanzadas, por ejemplo: 
- "La regresión, la variable 'profundidad' no se distribuye normal, pero la qqplot parece normal ¿Se cumple el supuesto de normalidad o no?" Estudiante de la licenciatura en Biología, diciembre 2017-enero 2018 (figura 3a).

- "Tengo una variable cualitativa y otra cuantitativa ¿cómo mido la relación entre ellas?" Estudiante de la licenciatura en Instrumentación electrónica, diciembre 2017-enero 2018 (figura 3b).

- "Si hay dos medidas de tendencia central (media y mediana) ¿Cuál ocupo?" Estudiante de la licenciatura en Economía, junio-julio 2017 (figura 3c).

- "El mapa del análisis de correspondencias se ve una mancha y no se distinguen los puntos ¿Cómo lo interpreto?" Estudiante de la licenciatura en Ciencias y técnicas estadísticas, enero 2019 (figura 3d).

Como se observa en la figura 3, la resolución de las preguntas que surgen del estudiante, debido al contacto con datos reales, hacen que el conocimiento se asimile de una forma más productiva y que la dinámica de la clase sea más participativa. El facilitador responde las dudas en clase y ante todo el grupo, posteriormente el estudiante al presentar su artículo final se ve obligado a registrar de manera escrita sus hallazgos (incluyendo sus dudas durante el proceso), con sus propias palabras (figura 3).

La resolución de problemas estadísticos en este sentido no es algo mecánico al estilo de una receta, como en los tradicionales cursos de estadística, sino que en realidad promueve que el estudiante al tener un problema (de su agrado) en mente, se ocupe de resolverlo y que adapte o descarte los temas vistos en clase para la resolución de su indagación personal. 


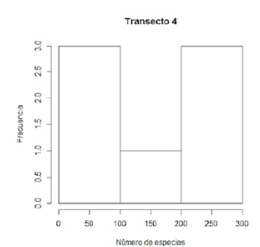

Fuente: Elaboracion propia usando R-project 3.3.2 La profundidad del ärea va incrementando conforme se acerca a la barrera arrecitin una minima de $36 \mathrm{~cm}$ en la orilla de la costa y un máxima de $90 \mathrm{~cm}$ cerca de la barrera arrecifal como se puede observar en el histograma 3. Est variable tambien influye en la posicion geografic en la que se encontraron los cridanios, debido a que necesitan luz solar al tener mina relación simbionte

Figura 2. Frecuencia de la profindidad en $\mathrm{cm}$

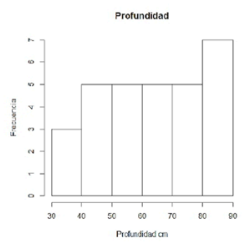

Fuente: Elaboración propia usando R-project 3.3 .2 Segín el analisis en el modelo de regresión lineal simple, la correlación con el método de Pearson entre la variable dependiente que es la riqueza de especies y la variable independiente que es

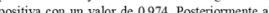

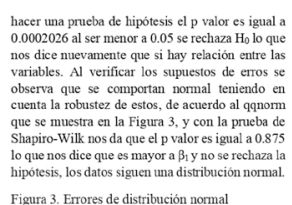

Figura 3, Errores de distribución norma

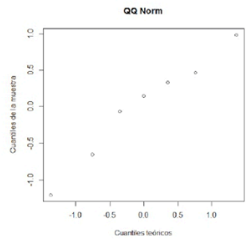

Fuente: Elaboración propia usando R-project 3.3 .2 Por último, si cumple con la homogeneidad de la varianza de errores seguin la Figura 4, lo que nos dice que la profindidad si influye directamente con la baja diversidad de cnicarios que presenta el arrecife rocoso de la Mancha, Veracriz.

Figura 4. Homogencidad en la varianza de crores

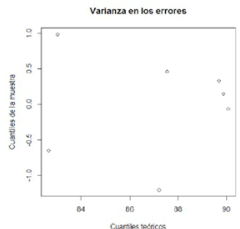

Fuente: Elaboracion propia usando R-project 3.3.2

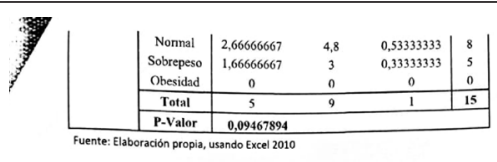

Al analizar P-Valor con respecto a las mujeres, se observa que este es menor a 0.05 , por lo tanto se rechaza la hipótesis plateada ( $\mathrm{HO})$ con un $99 \%$ de confianza.

Por el contrario, al analizar P-Valor respecto a los hombres, se observa que este es mayor a 0.05 , por lo tanto $\mathrm{H} 0$ no se rechaza y hay un $91 \%$ de confianza en el modelo

Para una mayor exactitud en los resultados se realizó un análisis de correlación simple, donde el principal resultado es el gráfico de puntuaciones de la figura 1.

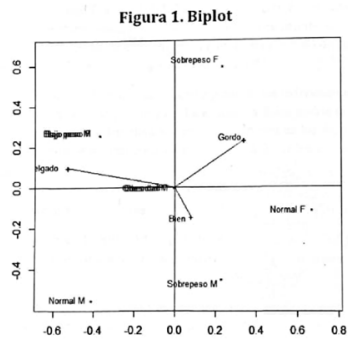

En donde el primer cuadrante muestra que las mujeres con sobrepeso se visualizan de forma correcta como "gordas".

En el segundo cuadrante de igual manera se observa que las personas delgadas se autoperciben corporalmente de forma real respecto a su IMC.

Extracto del trabajo final de la estudiante de la

Extracto del trabajo final de la estudiante de la Licenciatura en Biología, diciembre 2017-enero 2018. Licenciatura en Instrumentación Electrónica, diciembre 2017-enero 2018.

Figura 3. Extracto de los trabajos de los estudiantes de la EE ¿Cómo aplicar la estadística en proyectos de investigación?

Fuente: Elaboración propia. 
Desarrollo del pensamiento estadístico en estudiantes de nivel superior a través de una Experiencia Educativa

errores y los errores ajustados, donde se espera que los errores no tengan un patrón definido en su comportamiento.

Resultados

1. Análisis exploratorio.

En primer lugar las variables PIBP y PIBC tienen una diferencia de 0.00066 entre sus medias, mientras que la diferencia entre sus medianas es de 0.0003451 , es decit, la diferencia entre sus medias es mayor a la diferencia entre sus medianas (véase cuadroit). Por lo se cree que la mediana es una mejor medida de tendencia central para la comparación dé nivestras variables. En segundo lugar, otras de las diferencias en las variables son los valores minimos y máximos que se observan en cada una sas sicndo 0.0473276 el máximo valor de la variable PIBP contra un valor máximo de la variable PIBC de 0.0251748 (véase cuadro 1 ).

Con la información anterior, se sabe que la distancia existente entre la mediana y el valor máximo, de cada variable, es mucho más pronunciada en PIBP que en PIBC. Teniendo una idea de Ia distribuidas de manera más

\begin{tabular}{|c|c|c|c|}
\hline \multicolumn{4}{|c|}{ Cuadro 1. Medidas de tendencia central } \\
\hline Criterio & PIBP & PIBC & ADI \\
\hline Media & 0.0069455 & 0.0062855 & 7.145 \\
\hline $\begin{array}{l}\text { Media } \\
\text { Mediana }\end{array}$ & 0.0046079 & 0.0042628 & 7.530 \\
\hline $\begin{array}{l}\text { Medorda } \\
\text { Valor minimo }\end{array}$ & 0.0002557 & 0.0002158 & 2.120 \\
\hline \begin{tabular}{|l} 
Valor minimo \\
Valor Máximo
\end{tabular} & 0.0473276 & 0.0251748 & 9.820 \\
\hline $\begin{array}{l}\text { Dalor Mazinimo } \\
\text { distrancia entre Mediana-Minimo }\end{array}$ & 0.0043522 & 0.004046 & 5.41 \\
\hline 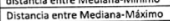 & $\begin{array}{l}0.0043522 \\
0.0427197\end{array}$ & 0.020912 & 2.29 \\
\hline
\end{tabular}

Los gráficos que se presentan en la figura 1 observamos las distribuciones de cada una de las variables, Como ya se anticipó, la concentración de los datos de PIBP es hacia los valores pequeños de entre 0.00 y 0.01 con una cola cargada hacia los superiores a este rango, $y$ un valor atipico que pertenece al valor máximo. Similarmente, la variable PIBC presenta colas cargodas hacia valores superiores a 0.01 , pero con un valor atipico menos aislado. Por su parte, a variable ADI presenta una concentración más normal con una cola ligeramente cargada a los valores menores a 5 y con un valor atípico cercano a 2 .
Extracto del trabajo final de la estudiante de la Licenciatura en Economía, junio-julio 2017
2.3 Análisis de correspondencias

Figura 1. Mapa simétrico del anălisis de correspondencias múltiple.

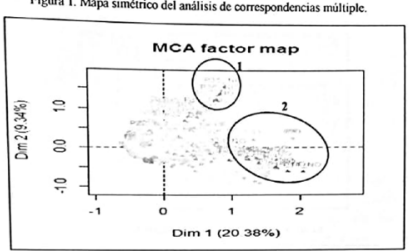

Fuente: elaboración propia con software $R$

Con el fin de resumir una gran cantidad de datos en un número reducido de dimensiones con la menor perdida de información posible se procedió a realizar un análisis de correspondencias múltiples el cual con tan solo dos dimensiones explica aproximadamente el $30 \%$ de la inercia de los datos. También se puede observar que el elipse 1 contiene las afirmaciones P35, P36 y P37 siendo estas las de mayor poder de discriminación. Dentro del elipse 2 se concentra un grupo de individuos los cuales son aquellos que en la mayoria de las

2.4 Perspectiva de los alumnos con respecto al emprendedurismo

Tabla 2. Respucstas a pregunta

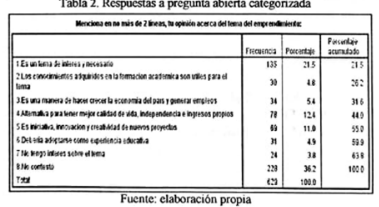

Finalmente se realizó un análisis textual para conocer la opinión acerca del emprendedurismo, se establecieron categorias de acuerdo a las respuestas obtenidas observando que la respuesta con mayor frecuencia es que encuentran el emprendedurismo un 4

Extracto de la primera retroalimentación de estudiante de la Licenciatura en Ciencias y técnicas estadísticas, enero 2019.

Figura 3 (continuación). Extracto de los trabajos de los estudiantes de la EE ¿Cómo aplicar la Estadística en proyectos de investigación?

Fuente: Elaboración propia. 
Resaltamos también que hemos encontrado que $8 \%$ de estudiantes que se destacan por su capacidad de autoaprendizaje y por cuenta propia indagan variaciones a las técnicas mostradas en clase (al menos un estudiante de cada curso intersemestral). En resumen, hemos registrado que 16 estudiantes han realizado preguntas que dan muestra de un grado de evolución en la comprensión de la aplicación de la estadística en la resolución de problemas de investigación, esto hace constar que hay evidencia de un $40 \%$ de los estudiantes que muestran el desarrollo de un pensamiento estadístico durante la participación en clase y en su reporte estadístico. Esto, no se da en cursos tradicionales en los que se trabaja con resolución de ejercicios estandarizados.

\section{CONCLUSIONES: ACIERTOS Y DIFICULTADES}

El valor educacional de usar experiencias de consultoría en la educación estadística es bien conocido (Sabo, 2016), la EE intersemestral ¿Cómo aplicar la estadística en proyectos de investigación? no solo planteó un escenario de consultoría, sino que permitió que los estudiantes inscritos adquirieran el conocimiento esencial de la aplicación de la estadística en su disciplina, que aprendieran de otras áreas y se interesaran en temas que no conocían. Esto amplía su visión del uso, aplicabilidad y necesidad de esta disciplina y despierte su curiosidad, que es uno de los elementos primordiales para el desarrollo del pensamiento estadístico.

Estamos de acuerdo con Hoerl y Snee (2017), en que cada día más se necesitan estadísticos holísticos que resuelvan problemas ágilmente, más aún se necesitan profesionistas de cualquier área de conocimiento que reconozcan y hagan uso de la estadística, por eso es por lo que los casos de estudio reales y no sacados de un libro o diseñados previamente, son necesarios para la educación actual. Que el estudiante proponga una pregunta de investigación y recolecte datos que no cumplen al pie de la letra con la teoría, los coloca en la necesidad de no solo conocer las técnicas y herramientas con las que cuentan para resolver el problema, sino de relacionarlas y/o combinarlas.

La construcción de un reporte estadístico a manera de un artículo científico, como evidencia de aprendizaje y logro de los dominios planteados, ha sido de los grandes retos a lo largo de estas tres aplicaciones. Hay una tendencia marcada a que los estudiantes provenientes del área Físico-Matemáticas, Biología y 
Química, Medicina y Ciencias de la Salud, tengan una mayor facilidad para la comprensión y redacción de esta evidencia de aprendizaje, a diferencia de los estudiantes provenientes de Humanidades y Ciencias de la Conducta, Ciencias Sociales y Economía, que presentan mayores dificultades.

El manejo de paquetería de software libre R-projectes una de las herramientas que más trabajo ha costado para la comprensión entre los estudiantes, es necesario ir más despacio de lo que originalmente se había planeado. Requiere realizar muchas prácticas en clase con la orientación del facilitador para que los estudiantes tengan la disposición de consultar sus dudas en la ejecución. Se ha detectado que con regularidad los errores que se cometen son: falta de paréntesis, falta de comas, la no distinción entre mayúsculas y minúsculas, errores de dedo al llamar a las variables, etcétera.

La presentación de su trabajo final ante la clase es una evidencia de aprendizaje muy valorada entre los estudiantes a lo largo de estas tres aplicaciones. Durante la misma, se les hizo notar las mejoras que pueden hacer, como, por ejemplo: dicción, expresión corporal, orden de las ideas, uso adecuado de las diapositivas, etc. Se exhortó a los estudiantes a participar en la mejora de los trabajos de sus compañeros, con ello no solo se apoyan entre ellos, sino que permite autoevaluarse y corregirse. Consideramos que este recurso contribuye en su formación integral universitaria.

Para responder a la pregunta de investigación ¿Qué resultados se observan como consecuencia de la implementación de las estrategias diseñadas para desarrollar el pensamiento estadístico? Acudimos a los resultados de este artículo: $40 \%$ de los estudiantes lograron la competencia de un pensamiento estadístico, 32.5\% la competencia de un razonamiento estadístico y $20 \%$ la competencia de una alfabetización estadística, por lo que podemos comentar que el curso fue exitoso. La pregunta que nos surge es ¿Por qué no hemos logrado $100 \%$ de éxito que es la meta a la que esperaríamos llegar? Tenemos algunas hipótesis que en futuros estudios tendríamos que demostrar: El carácter interdisciplinar de la materia hace que las habilidades de razonamiento de los estudiantes sean distintas debido a la disciplina de formación original; el ritmo de aprendizaje propio de cada estudiante; el hecho de que sea intersemestral, lo cual hace que todo sea más veloz; la familiarización con un software que va por comandos y no por ventanas, lo que lleva a una brecha disciplinar que no podemos acortar en un solo curso.

El enfoque que aquí se expone para desarrollar el pensamiento estadístico, parte de un curso completo de 60 horas/semana/mes y considera como centro 
la realización de un reporte estadístico, ya que el pensamiento estadístico no es medible a partir de tareas formuladas, aun cuando se enriquezcan con un contexto, así que requiere de la complejidad como su aliada. Hay estudios que prueban, a partir de ejercicios diseñados para ello, que entre las principales dificultades para resolver problemas de comparación de conjuntos de datos es que los estudiantes no los conciben como un conjunto, sino como elementos individuales (Orta y Sánchez, 2018). Estos autores proponen el contexto de riesgo, como uno de los elementos que modifica el razonamiento estadístico. Nosotros proponemos el reporte estadístico, como el elemento clave para desarrollar el pensamiento estadístico.

Y además de lo anterior, que tengan la oportunidad de dar sentido a sus conjeturas relacionadas con su disciplina con objetividad; explorar sus propias ideas; plantear hipótesis a partir de datos que sirven de base para una investigación y adaptar estrategias para argumentar sus conclusiones a través del uso adecuado de la estadística.

Por último, dejamos el comentario de un estudiante al finalizar el curso: "Me parece que esta experiencia educativa es fundamental para cualquier carrera ya que nos da una vista general de lo bueno que es emplear la estadística y nos ayuda para futuras investigaciones".

\section{REFERENCIAS}

Brown, E. N., y Kass, R. E. (2009). What Is Statistics? The American Statistician, 63(2), 105-110. https://doi.org/10.1198/tast.2009.0019

GAISE College Report ASA Revision Committee (2016). Guidelines for Assessment and Instruction in Statistics Education: College Report. American Statistical Association, (February), 1-61. http://www.amstat.org/education/gaise

Franklin, C. A., Bargagliotti, A. E., Case, C. A., Kader, G. D., Scheaffer, R. L., y Spangler, D. A. (2015). Statistical education of teachers (SET). http://www.amstat.org/education/ SET/SET.pdf

Franklin, C. A., Kader, G. D., Mewborn, D., Peck, R., Perry, M., y Scheaffer, R. L. (2005). Guidelines for Assessment and Instruction in Statistics Education (GAISE) College Report. Guidelines for Assesment and Instruction in Statistic Educaction (GAISE) Report. A Pre-K-12 Curriculum Framework. https//doi.org/10.3928/01484834-20140325-01

Garfield, J., DelMas, R., y Chance, B. (2003). The Web-based ARTIST: Assessment Resource Tools for Improving Statistical Thinking. Paper presented in the Symposium: 
Assessment of Statistical Reasoning to Enhance Educational Quality, (April). http:// apps3.cehd.umn.edu/artist/articles/AERA_2003.pdf

Gould, R. (2010). Statistics and the modern student. International Statistical Review, 78(2), 297-315. https//doi.org/10.1111/j.1751-5823.2010.00117.x

Hoerl, R. W., y Snee, R. D. (2017). Statistical Engineering: An Idea Whose Time Has Come? American Statistician, 71(3), 209-219. https//doi.org/10.1080/00031305.2016.1247015

Kuntze, S., Aizikovitsh-Udi, E., y Clarke, D. (2017). Hybrid task design: connecting learning opportunities related to critical thinking and statistical thinking. ZDM - Mathematics Education, 49(6), 923-935. https//doi.org/10.1007/s11858-017-0874-4

Orta, J. A., y Sánchez, E. (2018). Niveles de razonamiento sobre variación estadística de estudiantes de nivel medio superior al resolver problemas en un contexto de riesgo. Educación Matemática, 30(1), 47-71. https//doi.org/10.24844/EM3001.02

Rouquette-Alvarado, J., Suárez-Burgos, A., y Ariza-Gómez, E. (2014). Relevancia de la formación estadística en la universidad. La importancia de encontrarles sentido a las matemáticas. Reencuentro 69, 37-45.

Sabo, R. T. (2016). Providing Consulting Experiences Through Role Playing in a Graduate Statistics Course. Australian and New Zealand Journal of Statistics, 58(3), 319-333. https//doi.org/10.1111/anzs.12167

Secretaría de Educación Pública (SEP) (2011). Plan de Estudios 2011, Educación Básica. http://www.gob.mx/cms/uploads/attachment/file/20177/Plan_de_Estudios_2011_f.pdf Secretaría de Educación Pública (SEP) (2016). Propuesta curricular para la educación obligatoria 2016. http://www.gob.mx/cms/uploads/docs/Propuesta-Curricular-baja.pdf Secretaría de Educación Pública (SEP) (2017). Mapa curricular para la generación 20172020. http://www.dgb.sep.gob.mx/informacion-academica/mapas_curriculares.php

Tu, W., y Snyder, M. (2017). Developing conceptual understanding in a statistics course: Merrill's First Principles and real data at work. Educational Technology Research and Development, 65(3), pp. 579-595. https//doi.org/10.1007/s11423-016-9482-1

Tunstall, S. L. (2018). Investigating College Students' Reasoning With Messages of Risk and Causation. Journal of Statistics Education, 26(2), 76-86. https//doi.org/10.1080/1 0691898.2018.1456989

Universidad Veracruzana (1999). Nuevo modelo educativo para la universidad veracruzana lineamientos para el nivel licenciatura. http://www.uv.mx/meif/files/2015/03/ MEIF.pdf

Weiland, T. (2017). Problematizing statistical literacy: An intersection of critical and statistical literacies. Educational Studies in Mathematics, 96(1), 33-47. https//doi. org/10.1007/s10649-017-9764-5 
Web of Science (2018). Clarivate analytics.

http://apps.webofknowledge.com/WOS_Generalsearch_input. do;jsessionid=5A817342442BC2DFF64E54F5E1ECEC4C?product=WOS\&search_ mode $=$ GeneralSearch $\$ S I D=8$ DkL3UoieFQeP7I3EYZ\&preferencesSaved=

Diana Del-Callejo-Canal

Dirección Postal: Antonia Peregrino, 23. Colonia Badillo. C.P.91190, Xalapa, Veracruz, México.

Teléfono móvil: 2287535864 